\title{
Adverse Event Start Day
}

National Cancer Institute

\section{Source}

National Cancer Institute. Adverse Event Start Day. NCI Thesaurus. Code C83343.

The day of the week an adverse event began. 